\title{
Partage à la demande et clôtures : Quelques aspects des nouvelles maisonnées de Port-Vila
}

Demand-sharing and fences: Aspects of the new Port Vila household

\section{Knut Rio}

Traducteur : Christine Jourdan

\section{(2) OpenEdition Journals}

Édition électronique

URL : http://journals.openedition.org/jso/7780

DOI : $10.4000 /$ jso. 7780

ISSN : $1760-7256$

Éditeur

Société des océanistes

Édition imprimée

Date de publication : 15 décembre 2017

Pagination : 91-104

ISSN : 0300-953x

Référence électronique

Knut Rio, « Partage à la demande et clôtures : Quelques aspects des nouvelles maisonnées de PortVila », Journal de la Société des Océanistes [En ligne], 144-145 | 2017, mis en ligne le 15 décembre 2019, consulté le 15 mars 2021. URL : http://journals.openedition.org/jso/7780 ; DOI : https://doi.org/ $10.4000 / j s 0.7780$ 


\section{Partage à la demande et clôtures: Quelques aspects des nouvelles maisonnées de Port-Vila}

par

Knut RIO*

\section{RÉSUMÉ}

Cet article discute certains aspects de la vie urbaine dans la capitale du Vanuatu. Lors de mes dernières recherches de terrain (2010 et 2014), j'ai étudié l'économie des maisonnées et l'organisation sociale des quartiers informels existant autour de la ville de Port-Vila. Je présente ici un aspect particulier de ces quartiers et mets à l'épreuve le débat établi sur la réciprocité mélanésienne, soit le partage à la demande et le don. Dans le cas qui mintéresse, les résidents de nombreuses maisonnées installent un petit magasin où les membres de la famille et les amis du voisinage peuvent acheter les articles ménagers dont ils ont besoin. Comme la maison elle-même, ce petit magasin est clôturé et fermé, non par peur du vol, mais comme moyen de protection contre le partage à la demande agressif et la convoitise. Je défends lidée que les gens de Port-Vila ont recours à ces magasins pour protéger la valeur du partage de la famille et des voisins toujours plus envahissants

MoTS-CLÉs : Port-Vila, partage à la demande, économie marchande, quartiers d'habitat informels urbains

Les quartiers de Port-Vila (Vanuatu) sont devenus d'intéressants lieux d'expérimentation de nouvelles formes de socialité mélanésienne. Leurs formes s'adaptent constamment aux défis auxquels les ni-Vanuatu font face lors du passage de la vie rurale à la vie urbaine. Ici, les gens vivent dans des quartiers très peuplés, non seulement en cohabitation avec des gens auxquels ils seraient liés par des relations d'évitement au village, telles que relations entre affins et entre

\section{ABSTRACT}

This article addresses certain aspects of town life in the capital of Vanuatu. In my most recent fieldwork in 2010 and 2014 I have been investigating household economy and aspects of social organization in the settlements that rapidly spring up around the town of Port Vila. I will present one particular feature of these settlements as a test case for revisiting the long debate about Melanesian reciprocity, demand sharing and gift. Notably, in many households people set up a little store, from which kin and friends in the neighborhood can buy their household supplies. Like the household itself, the store is typically fenced off and barred in - not directly from fear of theft, but as a defense against aggressive demand-sharing and envy. My point will be that people in Port Vila now tend to use the store economy as a way of protecting the value of sharing from the too intruding world of relatives and neighbors.

Keywords: violences, Port Vila, demand-sharing, store-economy, urban settlements

sœurs et frères, mais aussi relations avec des gens originaires d'autres endroits, parlant d'autres langues et respectant d'autres façons de vivre (patrilinéarité/matrilinéarité, autres règles de mariage, autres formes de magie ou de sorcellerie, etc.). Comme les auteurs de l'introduction de ce volume l'indiquent, les gens du Vanuatu n'ont qu'une expérience relativement récente de la vie urbaine ; pour la plupart d'entre eux, l'indépendance (1980) fut le point de rupture

* Université de Bergen, Knut.Rio@uib.no

Traduit de l'anglais par Christine Jourdan, Université Concordia 
le plus important. Jusqu'alors, les gens des îles et des zones rurales périphériques pouvaient se rendre à Port-Vila ; ils pouvaient aussi travailler temporairement dans les plantations avoisinantes ou encore obtenir des emplois dans la fonction publique (police, hôpitaux ou bureau de poste). Mais une fois remplies leurs obligations envers les autorités coloniales ou administratives, ils étaient renvoyés dans leurs îles d'origine (Rousseau, ce volume, pp. 37-50). Port-Vila est le centre administratif du Vanuatu, pays indépendant depuis maintenant trois décennies. Même si les habitants ont réussi à s'approprier la ville, en quelque sorte ${ }^{1}$, l'adaptation à la vie urbaine et ce qu'elle implique demeurent aujourd'hui une question sociale et politique importante. Les citadins parlent constamment des coûts et des avantages liés au passage d'un mode de vie basé sur une agriculture rurale à celui basé sur le travail salarié dans la construction ou le secteur des services ; et ils parlent également du passage de la vie vécue dans un environnement social dominé par la présence d'un vaste système de parenté où les denrées agricoles circulent facilement, à une économie dominée par la rareté des biens de consommation et de l'argent.

Dans cet article, et en abordant un aspect bien particulier du transfert des valeurs rurales à la situation urbaine, je participe à une discussion qui porte sur ce que vivre dans une ville mélanésienne veut dire. Pour ce faire, je traiterai de la pression exercée sur la maisonnée urbaine et m'intéresserai aux stratégies mises en place par les citadins pour résister à cette pression. Ces stratégies consistent surtout en une transformation de la maisonnée en unité fermée et autonome, et ici, je mettrai l'accent sur quelque chose de plus particulier, soit les magasins domestiques installés par les citadins dans ou près de leurs maisons. Ces magasins sont créés pour répondre aux besoins de la famille immédiate et à ceux des voisins. Je suggère que ce genre de magasin répond à un dilemme difficile : d'une part, perpétuer l'idée du partage entre les membres de la famille et entre amis et, d'autre part, éviter l'épuisement financier des maisonnées que provoque le prélèvement imprévisible de nourriture et de biens de consommation. Quand les gens de Port-Vila parlent de cette forme dangereuse de déplétion de leurs ressources, ils utilisent l'expression bislama stikim nek (littéralement: " percer le cou »), indiquant ainsi l'angoisse qu'ils ressentent face à un partage à la demande agressive. L'expression s'applique au problème créé par la famille étendue mais aussi par les visiteurs venant des zones rurales, et à leur tendance à vider les provisions de la maisonnée sans fournir quoi que ce soit en échange.
Je considérerai ce problème sous l'angle d'une discussion anthropologique du partage à la demande (demand sharing) (Peterson, 1993) et essaierai de comprendre comment cette idéologie largement répandue peut s'adapter ici au contexte social très différent de l'économie de marché basée sur l'achat et la vente. Chez les Aborigènes australiens, comme l'a expliqué Peterson, le partage à la demande est basé sur des obligations résultant de rôles et positions dans des relations de parenté préexistantes. Ce système de droit et d'obligation de partage, qui ne fait aucune référence à la générosité en tant que telle, se manifeste, en pratique, lorsque les gens prennent ou demandent des choses à des proches. Je conçois qu'il faut faire preuve de prudence quand on importe un tel concept issu d'un autre contexte, mais je suis d'avis que le partage à la demande est également un concept utile pour parler de l'éthos généralisé du partage en Mélanésie. La question ici est de voir comment les conditions urbaines de pénurie affectent l'institution du partage à la demande, alors que les maisonnées ne peuvent plus continuer à faire face aux demandes et aux prélèvements incessants que les liens familiaux, d'amitié et de voisinage devraient idéalement permettre. Il me semble qu'une expérience de vie urbaine à Port-Vila consiste à réussir à préserver le « partage » et à éliminer la « demande ». Le magasin domestique est un bon point de départ pour changer les choses.

\section{Magasin domestique et construction de clôtures dans les quartiers d'habitat informels}

Cet article est basé sur des recherches de terrain menées à Port-Vila entre les années 2000 et 2014 de façon intermittente. Avant cela, Annelin Eriksen et moi avions fait du terrain dans une zone rurale du nord d'Ambrym (Rio, 2007 ; Eriksen, 2008). En nous déplaçant à Port-Vila, nous avons continué nos recherches de terrain grâce à nos contacts parmi les gens d'Ambrym qui vivaient maintenant en ville. Cela nous a amenés dans différentes parties de la ville, mais surtout dans les quartiers de Pango, Ohlen, Freswota, Ifira, Man Ples, Tagabe et de Bladiniere. Nous y avons passé la plus grande partie de nos journées : bavardant avec les gens assis dans leurs jardins, allant parfois dans des bars à kava l'après-midi, et parfois aussi à l'église avec nos amis d'Ambrym et leurs familles par alliance originaires d'autres îles. Cette recherche de terrain a eu lieu parmi les classes populaires de Port-Vila ; cela comprenait surtout des travail-

1. Le centre-ville de Port-Vila et les zones limitrophes de la côte sont, dans une large mesure, possédés ou loués par la population expatriée. Pour une grande part, les modèles d'emploi, de classe sociale et de logement qui prévalaient pendant l'époque coloniale existent encore. 
leurs non qualifiés conduisant des autobus ou des taxis, employés dans la construction ou dans le secteur du service, mais aussi des flâneurs sans emploi qui vont et viennent à Port-Vila, ainsi que des villageois d'Ambrym qui arrivent en ville par flots ininterrompus pour vendre leurs sculptures en bois dans les marchés d'artisanat et aux marchands venus de Nouvelle-Calédonie. Ceci signifie que nous avons surtout vu des maisonnées en situation constante de précarité et d'incertitude.

La situation des maisonnées urbaines ni-Vanuatu est telle que le revenu des salaires n'est pas suffisant pour permettre de vivre en ville. Un sondage informel effectué par le Vanuatu National Workers Union (Syndicat national des travailleurs de Vanuatu) en 2010 estime que le coût mensuel de la vie pour une famille de quatre personnes à Port-Vila est au minimum de 60000 vatus $(500 €)$. Le salaire minimum à Vanuatu, tel que fixé par le Minimum Wages Act de 2014, est de 170 vatus de l'heure $(1,4 €)$. Le salaire minimum procure un salaire net de 26000 vatus par mois (200€), ce qui correspond plus ou moins au salaire moyen gagné par nos amis d'Ambrym. Cela signifie plus directement qu'ils sont à court d'argent chaque mois pour couvrir les dépenses mensuelles telles que le loyer, les frais de scolarité, les transports, les vêtements et la nourriture, ainsi que les contributions aux cérémonies de mariage, de funérailles et de circoncision, et cela même si les deux parents travaillent. À cela s'ajoutent les frais occasionnés par les visiteurs temporaires provenant des îles qui, en général, participent moins aux frais de la maisonnée qu'ils ne consomment. Les stratégies utilisées pour combler ce déficit considérable dans le budget domestique incluent la vente de sculptures en bois, la participation à des cercles de microcrédit, le jeu dans les machines à sous, les emprunts à la famille, ou les demandes faites à la famille restée au village d'envoyer de la nourriture par bateau. Dans la vie quotidienne, le déficit et la pénurie se traduisent par une grande attention portée aux dépenses ; les pièces de 100 vatus (environ $1 €$ ) servent en général de base pour l'achat des marchandises vendues en magasin. Un paquet de sucre, quelques sachets de thé, du riz dans des petits sacs en plastique, de la viande ou du poisson en conserve, un pain, quelques bananes et un peu de chou des îles, quelques ailes de poulet, un trajet en bus, quelques cigarettes, une tasse de kava ou une recharge du téléphone portable sont autant d'articles qu'on peut se procurer avec les pièces de 100 vatus qui trainent dans les poches des gens.

Mais le problème rencontré par la plupart des citadins est que les rares occasions de dépenser se heurtent à une idéologie du partage. La situation en ville est à l'opposé d'une agriculture de subsistance qui fournit plus que nécessaire, au point qu'il y a toujours beaucoup de nourriture disponible et qu'il faille la partager et la manger avant qu'elle ne pourrisse. En ville, il y a toujours des pénuries d'argent et de biens de consommation; et puisque ces biens ne pourrissent pas, on n'a pas non plus besoin de les partager. Les gens soutiennent que la présence innovante des magasins domestiques leur permet de remédier à l'obligation de partager car le magasin existe pour être accessible et bénéfique à tous les membres de la maisonnée. Par contraste avec les autres commerces, le magasin domestique offre les biens de consommation en partage, mais ces derniers doivent être payés pour que la réserve de provisions puisse être renouvelée. La but de ce magasin n'est pas de produire un surplus : les clients trouveraient immoral que le magasin puisse être source de richesse pour le propriétaire.

Les maisonnées que nous fréquentons dans les quartiers informels de Port-Vila sont établies principalement sur des terres non règlementées comme celles des zones de Blacksands, Tagabe et Ohlen, toutes d'anciennes plantations coloniales. Une partie de ces plantations a été divisée en parcelles de terrain règlementées, comme celles de Freswota et Bladiniere, où les gens ont acheté des terrains dont l'infrastructure comprend l'adduction d'eau et l'alimentation en électricité. Cela ne change pas grand-chose aux conditions de vie et à la structure des bâtiments. En effet, comme cela arrive souvent, ceux qui construisent les logements ne sont pas ceux qui possèdent la parcelle de terrain en question. Ils saisissent cependant l'occasion de construire sur la parcelle de quelqu'un d'autre, ami ou membre de la famille. Même dans les zones règlementées, les gens n’investissent pas à long terme dans les maisons qu'ils construisent. Elles sont souvent fabriquées avec matériaux temporaires; elles ont un sol en béton mais des murs légers et des toits faits soit de tôle ondulée, de bâche, de bambou, de chaume de sagoutier, de carton ou de panneaux d'aggloméré.

Ces quartiers ont des allures de village, ne serait-ce que par les sentiers qui les traversent de façon organique, de maison en maison, et de telle façon que seuls les amis ou les membres de la famille peuvent trouver leur chemin entre les maisons. Les intérieurs de maison ressemblent à de sombres labyrinthes de pièces et de couloirs menant ici et là ; il n'y a qu'une seule entrée et très peu de sources de lumière. Ces habitations et leurs jardins sont entourés de fil barbelé et de clôtures faites en tôle ondulée. Ces clôtures mettent les maisons à l'abri des regards indiscrets tout en les protégeant contre l'extérieur.

De cette façon, les maisons sont protégées contre l'impression de danger associée à la vie urbaine. Elles prennent ainsi de plus en plus l'allure de hameaux qui se referment sur eux-mêmes. En raison de l'impression de danger et de prédation 


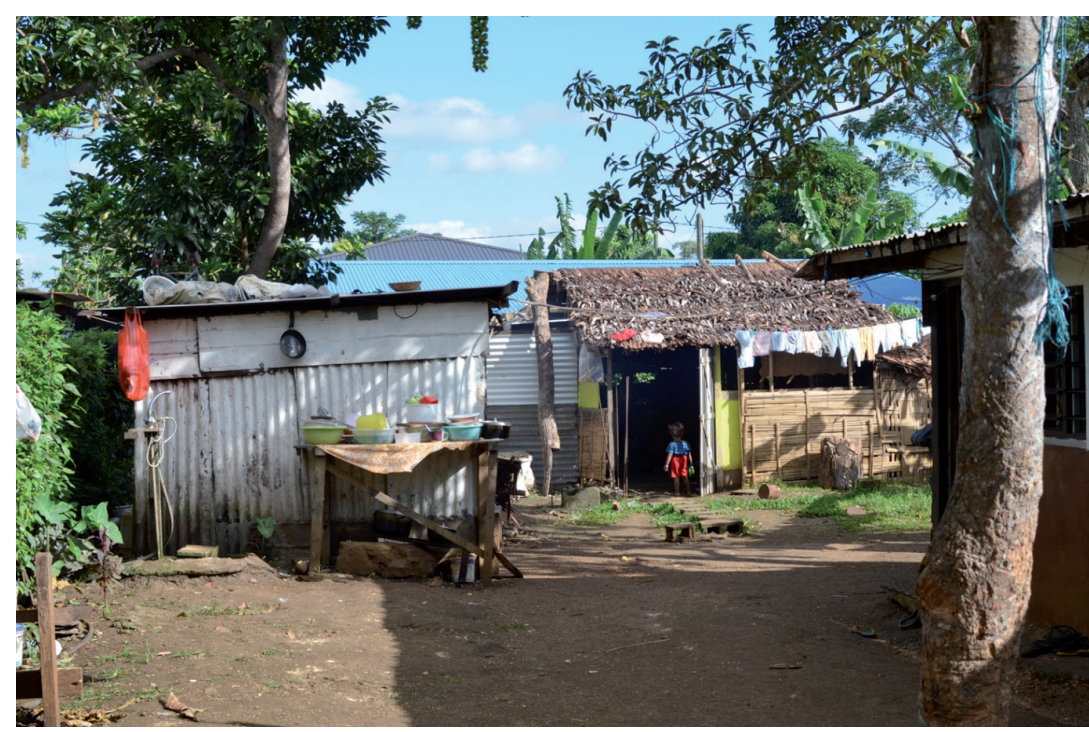

Figure 1. - Voisinage dans le quartier contrôlé de Freswota, 2014 (C Knut Rio) pas lieu d'habitude à l'intérieur des habitations, mais dans les endroits publics, à l'extérieur des discothèques ou des bars à kava, ou encore dans les rues la nuit quand les femmes rentrent seules chez elles. Un des problèmes auxquels les femmes urbaines doivent justement faire face est la perception qu'elles n'ont pas besoin d'être protégées; elles doivent plutôt se protéger elles-mêmes en s'habillant et se comportant correctement. Je pense que le fait de clôturer les maisons se

imminents qui règne hors de ces jardins, les gens mettent des cadenas à leurs portes et verrouillent leurs fenêtres. Les barreaux sont largement utilisés, spécialement dans les petits magasins et les bars à kava.

On peut être surpris devant cette augmentation de l'enclavement et de la sécurité dans un endroit comme Vanuatu. Gardons à l'esprit, qu'en comparaison avec Port Moresby et Honiara, Port-Vila est un endroit paisible où les vols sont peu nombreux. Le viol des femmes est par contre très répandu (Servy, ce volume, pp. 171184). Par exemple, en 2010, les détenus de la prison de Port-Vila se répartissaient ainsi : $60 \%$ avaient commis des viols ; $20 \%$ avaient commis d'autres formes de violence et seulement 3 ou $4 \%$ étaient coupables de vols ${ }^{2}$. Cela ne veut pas dire pour autant que les résidents de Port-Vila ne savent pas ce que les mots vol et effraction veulent dire : la situation prévalant à Port-Moresby leur fait peur. On peut donc se poser la question suivante: comment se fait-il que les gens de Port-Vila adoptent l'esthétique d'endroits aussi dangereux que Port-Moresby et se cachent derrière des verrous et des barreaux ?

Un élément d'explication se trouve dans l'inquiétude que les gens manifestent pour la sexualité et la sécurité des femmes. Un des facteurs importants est la nouvelle panique morale concernant la sexualité des femmes. Les femmes des maisonnées sont étroitement surveillées : leur façon de s'habiller; la modestie de leur toilette; l'absence de maquillage; le retour à la maison avant la nuit (Servy, 2017) sont contrôlés. Mais la panique morale et les préoccupations chrétiennes pour la pureté des femmes ne brossent pas un tableau complet. Le viol des femmes n'a rapporte davantage à d'autres processus sociaux quà la peur de la violence, du viol ou du vol. Cela s'inscrit plutôt dans une tendance à imposer à la vie sociale une dualité intérieur-extérieur. Le magasin domestique est un élément central de cette tendance car il cristallise un processus de transformation sociale qui tourne autour de quelques idiomes ruraux tels le hameau, le lignage et le partage entre membres du groupe.

Il ne fait aucun doute que le magasin domestique est un concept que les gens ont importé des zones rurales. Lidée d'avoir un magasin de village est d'abord apparue en lien avec les plantations pendant la période coloniale puisque les plantations étaient l'endroit où on pouvait se procurer des marchandises, du vin, du tabac et du sucre. Depuis lors, l'idée du magasin de village n’a pas changé, au point que chaque village du pays en contient quelques-uns. Dans un cas du nord d'Ambrym que je connais bien, les magasins desservent les différentes parties du village, conformément aux divisions lignagères et autres principes d'appartenance. Le magasin offre l'occasion d'interagir avec des gens appartenant à d'autres alliances et divisions lignagères. Dans ce contexte, le magasin agit comme un idiome social inhérent aux valeurs sociales. Le but du magasin est de permettre aux membres de la famille et aux amis de se procurer les articles de première nécessité : il fait donc fonction d'organisme d'assistance sociale. Il est censé être un espace bienfaisant où les gens peuvent interagir sur un pied d'égalité et se procurer des produits libres de toute obligation d'échange, de risque de sorcellerie ou de jalousie. Si vous achetez une boîte de corned-beef pour votre laplap (pouding) du dimanche, vous pouvez être sûr

2. Étude des infractions commises par les détenus (Département des services correctionnels, 2010). 
que cette conserve ne contiendra ni microbe ni poison qu'un sorcier aurait pu y mettre. Les magasins de village se procurent leurs marchandises dans les magasins en gros tenus par les Chinois de Port-Vila ou de Santo. Les valeurs de propreté sociale s'appliquent à tout ce réseau commercial et donc aux marchands chinois qui, en tant que parfaits étrangers, sont sans danger.

De nos jours, on remarque que l'idée de magasin de village existe aussi dans les zones urbaines. En ville, chaque grappe de maisonnées construit ses propres magasins sur ses terrains. Il existe ainsi maintenant à Port-Vila différents types de magasins qui varient selon les sphères sociales. On trouve d'abord au centre-ville des galeries marchandes et des supermarchés comme Au Bon Marché. On trouve ensuite les magasins chinois, soit dans le quartier chinois, soit en dehors du centre-ville. On y vend surtout des conserves, du thé, du sucre, du kérosène, des sacs de riz, mais également des ustensiles de cuisine et des outils de jardinage, du tissu et des vêtements, de l'équipement de pêche et de plongée et des jouets pour les enfants. L'inventaire de ces magasins fournit ainsi la partie la plus importante des matériaux nécessaires à la vie dans les villages ruraux, y compris pour le jardinage, l'alimentation et les cadeaux cérémoniels. Et, finalement, on trouve les magasins situés dans les quartiers informels : leur inventaire est exactement le même que celui trouvé dans les magasins chinois, mais les prix sont un peu plus élevés (de quelques centimes). Plusieurs d'entre eux sont tenus par des ni-Vanuatu et non par des hommes d'affaires chinois. Finalement, de nombreuses maisonnées ont leur propre magasin, contenant un stock similaire, mais qui se limite en général aux produits les plus utilisés comme le pain, le sucre, le riz, les sachets de thé et de café instantané, du chewing-gum, des cigarettes et des conserves de viande. Ici aussi, les prix sont un peu plus élevés de quelques centimes. D'après ce que les gens disent, ces magasins ont été installés pour rendre service aux gens de ces quartiers, de la même façon que les magasins de village rendent service aux villageois, et leur éviter d'avoir à aller trop loin le matin pour chercher du pain ou du sucre. En ce sens, le magasin domestique est un espace social particulier qui cultive une atmosphère de partage au sein d'un groupe restreint de gens, mais c'est un partage organisé autour de l'échange de commodités au moyen d'argent. Ces magasins sont habituellement situés à l'intérieur de la maison ou près de la cuisine; c'est l'endroit où les membres de la famille iraient normalement prendre la nourriture appartenant au foyer. Cette marchandisation de l'échange a l'avantage d'éviter que des saignées meurtrières (stikim nek) soient faites sur son propre stock. Le magasin domestique est une forme de résis- tance contre les formes agressives de partage à la demande.

Par comparaison, Rodolfo Maggio (2014) explique que le magasin domestique est très courant dans les quartiers informels d'Honiara. Il les considère comme des activités génératrices de revenus :

«c'est le moyen le plus facile de faire un profit avec relativement peu de travail. Tellement facile que les magasins ont essaimé partout pendant la dernière décennie. "

Sur un trajet de 700 mètres, Maggio en compta vingt-cinq (2014: 97). Mais il note aussi que le profit est très petit, et qu'il faut tenir compte d'autres facteurs pour expliquer l'ampleur du phénomène. À mon avis, un profit vraiment substantiel irait à l'encontre de la raison d'être du magasin domestique. Il se doit d'être non lucratif, car on ne trouverait pas acceptable qu'un parent proche ou un voisin utilise ses relations familiales ou amicales pour gagner de l'argent.

Les gens de Port-Vila savent toujours précisément combien ils doivent payer certains articles. Ils connaissent en général le prix exact des choses et n'hésitent pas à traverser la ville pour payer 50 cents moins cher l'article qu'il leur faut. Ceci veut dire que les magasins domestiques doivent idéalement vendre leur marchandise au même prix que le prix courant. C'est vrai des conserves de poisson ou de viande, du riz préemballé, du café et des bouteilles de sirop. Mais pour faire un profit, l'astuce est de diviser les paquets et de vendre les articles à l'unité : sachet de thé, cigarette, riz dans des paquets plus petits, etc. Par exemple, si une boîte de thé Lipton de 100 sachets coûte 500 vatus (5 euros), on peut vendre un sachet de thé pour 10 vatus et ainsi faire un profit de 500 vatus par boîte. Une boîte de 200 cigarettes se vend pour 5000 vatus, mais en vendant les cigarettes à l'unité 50 vatus chacune, on peut réaliser un profit de 5000 vatus (50 euros). Mais comme ces articles se vendent en petites quantités, il faut des mois pour vider ces boîtes. Un petit magasin que j'ai étudié en 2014 vendait chaque jour en moyenne cinq sachets de thé avec un profit de 5 centimes d'euro par sachet; cinq cigarettes avec un profit de 25 centimes d'euro par cigarette ; une bouteille de sirop avec un profit de 50 centimes par bouteille; un sac de sucre avec un profit de 50 centimes par sac; quatre sacs de riz de 100 grammes avec un profit de 10 centimes par sac; une boîte de thon en conserve avec 50 centimes de profit. Parfois, le magasin pouvait vendre un bocal de café instantané avec un profit de 50 centimes et quelques fruits avec un profit de 10 centimes par fruit. En moyenne, et sur une période d'un mois, le profit quotidien s'élevait à environ $3 €$, et le revenu mensuel à $90 €$ (9000 vatus). Mais j’insiste surtout ici sur 
le fait que le profit économique n'est pas le moteur du magasin. Étant donné que les marchandises qui quittent le magasin servent souvent à la consommation de la famille immédiate du propriétaire, on comprend qu'aux yeux de la femme qui le gère, de son mari, de ses enfants et de la famille en visite, le magasin sert surtout de moyen de contrôle de la consommation du groupe familial. Le magasin se trouve être un moyen très ingénieux pour contrôler la consommation immédiate de la famille étendue.

Alors que les maga-

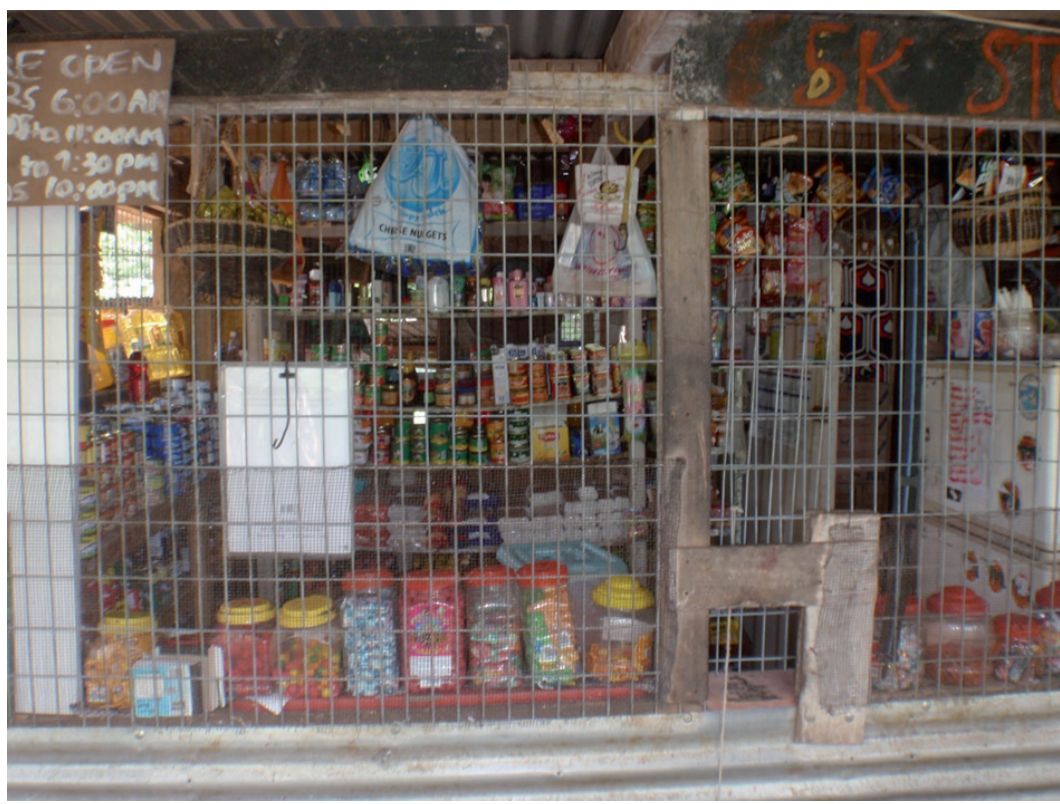

Figure 2. - Un magasin domestique desservant la banlieue immédiate près de Radio Ohlen, 2010 (@ Knut Rio)

sins domestiques sont

le théâtre de la consommation familiale et non du profit, les magasins chinois sont le théâtre du contraire. Ils servent de cadre à la production de profits importants mais non de rapports sociaux étroits. Les ni-Vanuatu n'ont que très peu de rapports avec la population asiatique de PortVila, si ce n'est qu'à titre d'employés. Les produits vendus dans les magasins domestiques sont achetés dans les magasins chinois; ils subissent une transformation en passant ainsi du domaine public au domaine familial. Et même si les marchands Chinois engrangent tous les profits - et ces marchands gagnent beaucoup d'argent grâce à la population urbaine pauvre - cela signifie aussi que les ni-Vanuatu peuvent protéger leurs proches contre la sphère du commerce.

Ces dernières années, les magasins domestiques se sont vus être associés à l'idée de "prospérité ", mais d'une façon spéciale qui n'a rien à voir avec le profit ni avec le partage au sein des relations familiales, mais plutôt avec le fait que le magasin est propre, bien tenu et bien géré. Ces qualités tendent vers un futur imaginaire où la maisonnée aura comblé le fossé qui existe entre les magasins et le marché. Écrivant sur Honiara, Maggio explique clairement que les magasins domestiques ne sont pas censés rapporter de l'argent, mais qu'ils servent à éviter que quelqu'un d'autre s'approprie cet espace commercial. Un de ses informateurs lui a confié :

"C'est juste une façon de nous assurer que l'argent reste à l'intérieur de la maison. » (2016:9)

Et Maggio d'expliquer de manière convaincante que finalement, et spécialement pour les gens convertis à l'Église pentecôtiste à Honiara, ces adaptations économiques de la maisonnée à la sphère du marché permettent aux habitants des villes d'échapper à la "tyrannie du besoin " (2014 : 259) causée par l'économie informelle, la pauvreté et la précarité causée par la pénurie. Parlant des Kwara'ae de Gilbert Camp (Honiara), Maggio conclut que :

"dans des conditions de pauvreté, des tensions apparaissent entre la valeur attribuée au partage et le besoin de joindre les deux bouts, tensions que les Kwara'ae tentent de résoudre par de l'argent et de la nourriture. » $(2014: 24)$

Ce qu'il appelle une "économie morale domestique " (2014: 90) devient très intéressante car la distribution de biens propres aux relations de partage, dès lors qu'elle se fait par l'intermédiaire du magasin domestique, se rapproche de ce que nous considérons normalement comme des relations propres au marché. Nous devons considérer cependant que si cette économie morale domestique prend la forme de ce type de relations, c'est parce qu'elle procure aux citadins un avantage quelconque. L'idée de "garder l'argent au sein de la maison " représente avant tout une manière de penser à la famille proche et au voisinage urbain, plutôt qu'une façon de penser à l'argent en tant que tel - puisque de toute façon, l'argent aboutit dans les poches du marchand chinois. Les magasins domestiques sont un moyen de résoudre les dilemmes urbains : ils créent un espace où les gens peuvent se rencontrer entre les maisons, ils approvisionnent les amis et la famille en denrées de première nécessité, et tout cela à l'intérieur d'un 
espace protégé des prélèvements et des abus. Le fait que de nombreux magasins arborent des panonceaux sur lesquels on peut lire no kaon! (pas de crédit!) témoigne de la tension qui existe entre le désir d'entretenir des relations amicales, mais pas trop quand même.

Je soutiens l'idée qu'on assiste ici à une transformation du magasin de village servant d'aide sociale à la famille étendue dans un contexte d'abondance, en un magasin de quartier urbain permettant de gérer le partage à la demande en situation de pénurie. Je pense également que la construction de clôtures autour des maisonnées et l'installation de magasins domestiques sont étroitement liées.

On peut s'étonner que les Mélanésiens souhaitent voir leurs proches s'acheter mutuellement de la nourriture. Et on peut être intrigués qu'ils aient des réactions paranoïaques à propos de la sécurité. Ces problèmes sont liés au type de formation sociale qui se développe dans ces contextes urbains. Mon approche s'inscrit dans la continuité du précepte de Polanyi (1957), à savoir que toutes les formes économiques sont instituées sur des formes sociales données. Par conséquent, ce que nous tendons à qualifier de marché, incluant l'argent et le commerce, fait partie de processus historiques particuliers qui se sont institués lors de circonstances sociales et historiques particulières (Polanyi, 1957). Il en découle que le fait d'acheter et de vendre n'est pas en soi un phénomène unitaire qui peut se comprendre indépendamment des structures sociales dans lesquelles il a lieu. Les relations sociales afférentes aux marchands chinois de PortVila, qui rappellent le personnage bien connu du "négociant étranger " (Polanyi, 1957 : 260), sont très différentes de celles afférentes au magasin domestique. Mon impression, corroborée par les travaux de Maggio à Honiara, est que les relations de marché présentes au sein de la maisonnée sont établies pour résister et rompre d'une part, avec les relations impersonnelles typiques de ces négociants étrangers, et d'autre part, avec les formes agressives de partage à la demande qui sont vécues comme des relations sociales intrusives.

\section{Le partage à la demande s'apparente à la générosité et non à l'échange}

Le concept de partage à la demande, aussi conçu comme du "vol toléré "(tolerated theft) (Peterson, 1993: 861), est apparu au cours des dernières années comme un concept qui a changé notre vision des sociétés de chasseurscueilleurs (Peterson, 1993; Woodburn, 1998 ; Riches, 2000; Jimenez et Willerslev, 2007; Widlok, 2013). Le partage à la demande fait référence aux droits mutuels qu'ont les gens liés par des relations de parenté déterminées, de prendre et de faire usage mutuellement des biens et possessions de l'autre. Tout en critiquant une perspective "noble sauvage " appliquée à la valeur du communautarisme et de la générosité, ces auteurs mettent plutôt l'accent sur le " prélèvement" (taking) que ce partage implique. Il est maintenant clair que le fait pour quelqu'un de demander de la nourriture ne s'apparente pas à un appel moral à la générosité ; il s'agit plutôt d'une technique ou d'une aptitude acquise indépendamment de considérations morales. À l'inverse de la tromperie ou de la duperie, le fait de "prendre » n'implique pas de discussions éthiques ou de conséquences et sanctions morales (Peterson, $1993: 868$ ).

On peut, sans exagérer, transposer ces leçons venant de situations propres aux sociétés de chasseurs-cueilleurs à la valeur attribuée au partage au Vanuatu. Dans l'économie villageoise d'Ambrym, les gens cultivent des légumes et des fruits et élèvent des cochons. Au sein d'un lignage ou d'un hameau, les gens travaillent ensemble et partagent le produit de leurs efforts. Ceci se passe à l'intérieur d'un groupe de hameaux organisé selon une descendance patrilinéaire. En dehors de cette sphère, et dans l'économie cérémonielle du mariage, de la mort ou de la circoncision, les groupes de hameaux font des échanges avec des groupes de hameaux similaires. Cela concerne d'abord l'action de "donner la vie". Le groupe de la famille de la mère (wuruen) fournit la "vie " sous forme de sang matrilinéaire, de dons de cochons vivants, de légumes, de nattes de couchage ou de nattes vestimentaires. Il s'ensuit que les gens qui reçoivent cette succession de dons de vie doivent rétablir ce déséquilibre hiérarchique en remboursant le "le don de vie " par le sacrifice de cochons et l'offrande d'argent. Par l'intermédiaire de ces cérémonies, ils remplacent l'idée du don par l'idée de l'échange, substitution attestée dans le vocabulaire quand le mot "don " est remplacé par le mot "paiement " (Rio, 2007b). Autrement dit, il n'y a pas que dans les villes que les choses s'achètent. À mon avis, les résidents de ces zones rurales ont depuis longtemps utilisé le mot "paiement" (pem en bislama) comme une expression idiomatique qui crée l'autonomie de la personne. Les paiements cérémoniels de masques et de savoirs secrets dans la société rom, les paiements faits pour obtenir les droits sur des modèles de sculptures tels que les tambours atingting à deux ou trois faces, ceux faits pour l'obtention d'articles symboliques particuliers comme le miroir ou le peigne utilisés lors des cérémonies d'initiation yengfah pour les filles, le paiement de parcelles de terrain pour y construire une maison, ou même le fait d'insister pour payer le lait en bouteille pour un bébé qu'on adopte, tout cela constitue 
des paiements institutionnalisés qui permettent à l'individu de se libérer d'obligations sociales et d'obtenir un pouvoir d'action autonome et une capacité d'échange égalitaire avec d'autres. Cette façon de penser remonte au moins à une centaine d'années, quand W.H.R. Rivers signalait la présence de paiements similaires, et mettait l'accent en particulier sur le fait que lors de leur circoncision, les garçons devaient "payer " leur oncle maternel pour obtenir le droit de voir et de posséder leur propre pénis. Rivers, qui a observé les cérémonies d'initiation masculine dans la partie occidentale de l'île d'Ambrym en 1914, faisait la remarque suivante :

«Sur Ambrym, on ne veut pas que le gland du pénis soit vu... mais non à cause d'une crainte quelconque de la magie ou à cause d'une peur liée à autre chose, mais parce que les gens ont payé pour voir le gland lors de la cérémonie pakvi. Ils refusent que celui qui n'a pas fait ce paiement puisse voir ce que eux-mêmes ont payé. »3 (Rivers, in Layard, 1942 : 478).

Dans la même ligne de pensée, on remarque que la pratique maintenant appelée taoboan ou "abattage de cochon» sur Ambrym consiste pour les hommes à abattre des cochons tout au long de leur vie à titre de paiements faits aux membres croisés de leur famille, idéalement des deux côtés, c'est-à-dire à ceux de leur famille maternelle et à ceux de la famille de leur épouse, pour qu'à la fin de leur vie ils puissent avoir le droit sur eux-mêmes - non seulement de voir leur pénis - mais également le droit de percevoir leur personne entière comme étant autonome de tous les hameaux environnants, le leur excepté.

Cette façon de penser est ancrée dans la vision du monde prévalant sur Ambrym; le résultat, similaire sans aucun doute à ce qu'on voit dans les autres îles de l'archipel, est une insistance généralisée sur le paiement lorsqu'il s'agit de droits, d'autonomie et de stature individuelle. Le statut obtenu par le paiement ne peut pas être partagé, mais ne peut pas non plus être repris. Il faut cependant garder à l'esprit que cette autonomie se définit vis-à-vis des autres lignages qui ont produit l'individu et vis-à-vis de son propre lignage. Tout le long de sa vie, un individu cherche à établir un équilibre et une autonomie par rapport aux membres des autres lignages, qui ne sont pas comme lui. Cet effort visant à obtenir égalité et autonomie de l'individu est constant puisque les autres lignages maintiennent tout au long de leur vie leurs propres activités de dons de vie et continuent à revendiquer leur participation à sa propre vie et à celle de son lignage. Cela fait partie de l'élaboration permanente des rapports entre les lignages d'Ambrym.
Par contraste, au sein d'un lignage (buluim), et au sein de la fraternité du groupe de pairs (bulbulan), l'individu ne fait qu'un avec le lignage luimême. Sur Ambrym, il n'est pas important de devenir individualisé ou autonome vis-à-vis du lignage, puisqu'en fait le lignage et la personne ne font qu'un. Il est donc complètement naturel de partager au sein de son hameau et de son lignage les produits nécessaires à la subsistance, les biens de consommation, ou les produits du potager, sans référence aucune à des considérations d'ordre moral. Comme dans le cas des sociétés de chasseurs-cueilleurs évoquées plus haut, ce partage se manifeste par le prélèvement ; ceux qui ont des droits de partage peuvent aller prendre ce qu'ils veulent dans un hameau sans se soucier de questions morales ou de sanctions. Dans la vie quotidienne, les membres d'un lignage partagent la nourriture et il n'est pas rare de voir des gens prendre de la nourriture, des cigarettes ou des vêtements dans les maisons des autres. Il ne s'agit pas ici de donner des choses, mais bien de prendre selon la philosophie du partage à la demande. Au cours du temps, les gens ont trouvé des manières détournées pour éviter que leurs biens soient ainsi emportés. Sur Ambrym, le risque de l'envie entre dans les relations lignagères comme un poison. Si quelqu'un refuse de mettre en circulation des biens dans le hameau, les rancunes à son égard risquent de s'installer au sein du lignage, rancunes qui peuvent causer la maladie et même la mort de la personne égoïste. On pense qu'il ou elle sera victime d'abiou, ce que nous appelons la sorcellerie ou la magie (Rio, 2002, 2018). On blâmera les membres du lignage ou du groupe de pairs. Nous notons qu'il s'agit là d'un danger inhérent aux relations internes du hameau et non aux relations entre les villages. Cet aspect de l'abiou, en tant que difficulté rencontrée par les lignages, se révèle quand le partage à la demande interne au hameau se heurte à des obstacles. Ceci est d'autant plus problématique que ces obstacles ne peuvent pas être surmontés par des échanges cérémoniels, comme c'est le cas lors des disputes entre lignages. Ces problèmes de sorcellerie demeurent un élément pénible de la vie interne du hameau.

Tournant notre regard vers la forme que prennent les quartiers urbains de Port-Vila, nous devons garder à l'esprit tous les aspects concernant le paiement, les droits, l'autonomie, le prélèvement, la sorcellerie et les relations intérieuresextérieures dont je viens de parler. Après tout, les gens qui habitent Port-Vila gardent encore un ancrage distinct dans les zones rurales du pays. $\mathrm{Au}$ Vanuatu, et c'est le point le plus important ici, le prélèvement et le partage à la demande ne

3. "There is an objection in Ambrym to the glans penis being seen, but [...] this is not due to the fear of magic or fear of any other kind, but it is because they have paid for the exposure of the glans in the pakvi ceremony. They object to anyone who has not so paid seeing what they have paid for. " 
constituent pas un modèle généralisé d'échange. Ils ne fonctionnent qu'à l'intérieur des paramètres restreints de hameaux où les gens se considèrent comme faisant partie d'un tout. De nombreux travaux ethnographiques portant sur le Vanuatu ont montré que l'appartenance et l'accès aux lignages, aux hameaux et aux groupes de parenté peuvent être très flexibles, au point que les individus vont et viennent très librement entre les hameaux. J'ai montré ailleurs (Rio, 2007) que pour comprendre les structures d'appartenance à Ambrym, nous devions penser en fonction des hameaux et non des lignages, les gens adaptant les termes de parenté en fonction de leur lieu de résidence. Sans aucun doute, la cohabitation est la variable associée au prélèvement et au partage à la demande. Ceux-ci sont institués sur l'idée que le lignage et le hameau partagent une substance commune qui donne à tous et à chacun le droit d'utiliser n'importe quel bien ou fruit du travail horticole disponible à l'intérieur du système de parenté et de mariage. Par contre, pem (le paiement) est basé sur les relations entre hameaux, au sein desquelles la valeur de l'autonomie et des droits est constamment établie par des échanges avec des groupes extérieurs. Quand le partage à la demande et le paiement existent au sein des maisonnées de Port-Vila, cela devient particulièrement intéressant.

Mais commençons par situer ce problème au sein d'une discussion plus large sur la réciprocité. L'aperçu que j'ai présenté plus haut sur la dualité du modèle de transactions - paiement et partage - se retrouve aussi dans les travaux d'Alfred Gell en Papouasie Nouvelle-Guinée (1992) et ceux de Joanna Overing en Amazonie (1992). Eux aussi établissent une distinction entre le partage et l'échange. Tous les deux soutiennent l'idée que dans ces sociétés, partager ce n'est pas du tout donner, et ce n'est pas non plus espérer ou s'attendre à un retour sur ce don. Quand on considère le partage à la demande, les trois obligations du modèle théorique de Marcel Mauss (donner, recevoir et rendre) s'étiolent, comme le fait la personne individuelle puisque le partage se résume à la manipulation d'un ensemble de substances de vie mutuelles, comme la nourriture, le sang, le sperme, les vêtements, etc. D'après Gell, cela a été une erreur de classer la circulation de ces biens sous la rubrique générale de la réciprocité, comme l'a fait le modèle de Sahlins sur la réciprocité généralisée, où il y a confusion entre échange de dons et partage (Sahlins 1972). Comme le fait remarquer Gell, ce que Mauss considérait comme un don était en fait calqué sur certaines formes rituelles de dons (la kula et le potlatch en particulier) qui impliquaient la rencontre cérémonielle avec d'autres personnes importantes, d'autres lignages et d'autres tri- bus. Gell était convaincu que dans la Mélanésie ancienne, ces dons concurrentiels étaient des phénomènes marginaux, partie prenante de relations rituelles avec des gens situés à la périphérie de leur propre horizon social. Il s'opposa à Mauss et Sahlins en disant que les rituels d'échange de dons ne sont pas la base de l'établissement de relations sociales proches, mais que le partage l'est. Sur un axe qui lierait les relations proches aux relations distantes, l'échange rituel de dons et les transactions marchandes se situent du côté des relations distantes, alors que le partage à la demande se situe du côté des relations proches et intra-lignagères. Les données de Gell sur les Umeda suggèrent que ces derniers ressemblent aux chasseurs-cueilleurs en ce qu'ils pratiquent le partage au lieu de, ou du moins plutôt que, la réciprocité. Ce principe est très fortement suggéré par le titre de l'article de James Woodburn:

"Le partage n'est pas une forme de réciprocité. " (1998, souligné par moi)

Le potlatch, le moka et la kula ne sont pas des situations prototypiques: ce sont des cas périphériques d'échange de commodités, transformés en compétition rituelle lors de conditions historiques particulières. En ce sens, l'argument présenté par Gell s'apparente aux commentaires de Polanyi sur le "négociant étranger ", à savoir que cet aspect serait présent dans de nombreuses sociétés anciennes d'où quelques personnes partiraient en expéditions commerciales pendant une partie de l'année (Polanyi, $1957: 260$ ).

Larticle d'Overing (1992) sur le partenariat commercial en Amazonie souligne la même chose. Les relations de troc entre hommes situés de part et d'autre de frontières culturelles porteraient sur des objets ayant une valeur d'usage, mais cette valeur d'usage dépendrait du pouvoir et de la puissance que leur auraient insufflé ceux qui les ont fabriqués. Comme dans le cas du don analysé par Mauss, ces objets troqués ou échangés seraient imprégnés de l'esprit de l'artisan et continueraient à garder leur puissance, leur dangerosité et leur utilité précisément en raison de ces bonnes relations à distance. Il serait erroné de penser que ces objets d'échange, ou l'échange luimême, soient impersonnels ou aliénés. Comme l'a fait remarquer Polanyi (1957 : 260) :

«Dans ces conditions, le commerce en général
n'existe pas."

Le commerce n'existerait donc que pour des objets particulièrement importants et signifiants et qui auraient une grande valeur symbolique en matière de reproduction et de régénération. Par contre, le partage à la demande met en jeu 
des objets qui sont importants pour la reproduction et la régénération, mais qui sont considérés comme des riens car il y en a en abondance. Selon le modèle de Gell, la reproduction de la société Umeda ne dépend pas de l'échange, mais bien de "l'obligation morale" (1992: 152) de remplir son rôle de mère, de frère, d'épouse ou de cousin. Les services rendus, les faveurs, la nourriture et les objets sont transmis selon des rôles sociaux prescrits; Gell les considère comme des relations " non réciproques » telles qu'elles existent entre parents et enfants ou mari et femme.

Il existe une vaste littérature portant sur les formes modernes de prélèvement ou de partage à la demande. On pense, par exemple, au système wantok en Papouasie Nouvelle-Guinée, aux envois de fonds (remittances), aux pratiques telles que la piqure $\left(\right.$ nilim $\left.^{5}\right)$ aux îles Salomon, ou à la saignée (stikim nek) au Vanuatu. Bien que la littérature ne les considère pas encore comme des formes de prélèvement ou de partage à la demande, ces formes témoignent cependant de l'étendue du système en train de se mettre en place en Mélanésie. Par comparaison avec le moka, la kula ou le malangan, ces pratiques sont sans lien avec l'individuation, l'autonomie ou la réputation d'une personne ou d'un groupe. Mais elles signalent à quel point les individus sont toujours sujets à être saignés et appauvris par les autres. Mais ces mots sont récents et surtout urbains, et ils reflètent les expériences de la vie urbaine de ces dernières décennies, exprimant d'abord et avant tout la forme que prend le partage à la demande quand l'abondance prévalant en milieu rural est remplacée par la pénurie en milieu urbain. Gardant à l'esprit l'argument de Polanyi selon lequel toutes sortes d'actions sont ancrées dans les structures sociales, nous pouvons nous poser la question suivante : qu'arrivet-il quand une société établie sur le partage à la demande se réoriente vers la consommation dans une économie de marché ? Je pense que nous avons ici un cas test de l'institution elle-même.

Dans ce contexte, je trouve très intéressant de pouvoir comparer la situation de la " Mélanésie ancienne » décrite par Gell avec celle de l'Amazonie contemporaine décrite par Joanna Overing. Comme Gell, Overing est très critique d'une littérature erronée qui a surestimé l'importance de l'échange et sous-estimé celle du partage. Mais son travail est encore plus utile pour le cas qui nous concerne ici car elle explique précisément comment la situation du village de Piaroa et le partage institutionnalisé fonctionnent dans une économie de marché basée sur l'achat de biens de consommation. Comme le fait Gell, elle décrit la dichotomie importante de la vie Piaroa, soit l'acquisition et la transformation des ressources par le travail et "l'échange " (palou) en tant qu'acquisition de biens créés par des personnes venant de l'étranger. Ceci existe dans un contexte politique de relations extérieures entre des partenaires commerciaux individuels, égaux mais en compétition. Les objets échangés sont puissants puisqu'ils sont traités rituellement par les fabricants ; on peut les comparer aux coquillages de la kula qui sont aussi des objets forts et puissants contenant l'esprit de la personne qui les a faits. Quand les Piaroa vont dans les magasins situés dans le bourg pour faire des achats, ils n'utilisent pas le terme d'échange. Overing signale que les produits de la forêt et des magasins sont traités différemment des objets étrangers, puissants et dangereux, obtenus par échange. Les relations avec les étrangers, conçues comme dangereuses, sont contrastées avec celles entretenues avec les membres appartenant à la communauté, conçues comme sécuritaires. Les Piaroa classent le fait de faire des achats dans la même catégorie que la chasse, mettent l'accent sur le prélèvement (taking) et considèrent qu'acheter s'apparente à la prédation. Les Piaroa en parlent comme s'il s'agissait "d'aller se promener pour voir ce qu'on peut trouver », un peu à l'instar des gens de Port-Vila qui disent mi wokbaot nomo ("je n'ai fait que me promener ") quand ils racontent ce qu'ils ont fait pendant leur journée en ville. Dans la société Piaroa, la place du marché est un endroit où l'on peut fouiller pour trouver ou ramasser quelque chose. Par contre, le commerce et l'échange des objets prestigieux sont complètement périphériques au monde du marché car ils sont basés sur des relations individuelles entre hommes originaires de régions différentes qui s'échangent des biens qu'ils ont personnalisés. Pour les Piaroa, se promener en ville équivaut à se promener en forêt, les deux activités consistant en un ramassage aléatoire de ce qui se présente. Les biens de consommation ne sont pas identifiés comme appartenant à quelqu'un et ils ne sont pas, par essence, des objets spirituels ou puissants. Les magasins sont perçus comme sécuritaires et comme des endroits où les relations internes peuvent avoir lieu, c'est-à-dire des endroits où les membres proches de la famille peuvent se retrouver et se procurer les produits qu'ils aiment.

Mais retournons à la Mélanésie urbaine. Appliqué à la situation de Port-Vila, ce que j'énonce plus haut nous donne des clés pour penser le monde des magasins et du marché. On peut dire, comme le font souvent les gens de PortVila, que quand on vit en ville, on fréquente les magasins chinois comme on irait dans son jardin potager. Quand ils font cette analogie, les gens oublient que les jardins potagers sont source de 
toutes sortes de problèmes: disputes à propos des droits de propriété, vols, destruction par les animaux, mauvaises conditions climatiques, travail difficile, manque d'aide. Ils disent en fait que si l'on est dans un village et qu'on veut quelque chose à manger, il suffit d'aller au potager et de prendre ce quil faut pour la journée, légumes, noix ou fruits. Le potager est perçu comme un domaine d'interaction gratuit et bienveillant. De la même façon, le magasin est un endroit où les relations sociales sont égalitaires, anonymes et faciles. Les gens utilisent des termes de parenté respectueux (tante ou oncle) pour s'adresser aux commerçants. Ce faisant, ils prétendent que la capitale nationale est une extension de leur hameau à l'échelle nationale. Dans ces conditions, l'atmosphère qui prévaut quand on fait des achats se rapproche davantage de celle qui existe entre membres de la famille que de celle qui prévaut lors des échanges et sacrifices cérémoniels entre membres éloignés de la famille. Ce ne sont pas non plus des conditions au cours desquelles on peut obtenir une autonomie individuelle ou des droits personnalisés, puisque le concept de pem qui existe dans les zones rurales n'est pas pertinent en ville. Le fait d'acheter quelque chose ne nous donne pas de droit sur l'essence même de cet objet. Par exemple, le fait d'acheter une bouteille de coca-cola ne nous donne aucun droit sur le coca-cola en général, mais nous donne simplement le droit de boire la boisson contenue dans cette bouteille. Toutes les marchandises du magasin sont étalées devant nous ; n'importe qui peut acheter ce qu'il veut car les produits vendus dans les magasins chinois sont considérés comme des petits riens (samting nating) qui se consomment ou se cassent facilement. Mais bien sûr, tout le monde sait que ce n'est pas un endroit où on peut "prendre ». Les gens connaissent très bien le concept de vol qui prévaut en ville, c'està-dire un crime passible de punition. Dans les magasins, on ne peut prendre quelque chose que si on le paie. Comme je l'ai indiqué plus haut, les produits sont vendus en petites quantités: pour un peu de monnaie, on peut se procurer quelques petites choses. Le magasin est simplement un endroit où on peut acheter et vendre des choses simples et pas chères.

Port-Vila est devenue la capitale nationale après l'indépendance en 1980 . Il me semble que lorsque les gens ont commencé à s'y installer, ils ont conçu l'indépendance un peu comme s'ils s'appropriaient les plantations, c'est-à-dire comme s'ils pouvaient profiter des nombreux magasins et du marché du travail qu'elle procure. D'ailleurs, quand les gens des îles pensent à Port-Vila, ils l'associent à une grande plantation - ce qu'elle était d'ailleurs avant l'indépendance. La vision du mouvement indépendantiste était qu'une fois l'indépendance obtenue, les habitants du pays pourraient ensuite vivre tous ensemble sur les plantations. Ils pourraient ainsi se débarrasser de toutes sortes d'inégalités coloniales ou coutumières et par le fait même étendre à la grande ville la moralité du partage propre au lignage. Cette image colore encore aujourd'hui la façon dont les gens interagissent avec PortVila. D'ailleurs, si on leur demande de nommer les avantages de la vie en ville, ils parlent d'une part des produits disponibles dans les magasins - entre autres, le soda et la glace qu'ils peuvent y consommer, et les ailes de poulet qu'ils peuvent y manger - et d'autre part ils parlent du travail qu'ils peuvent y trouver. Mais ils parlent aussi d'une forme particulière de liberté, celle de pouvoir se promener partout en ville et d'acheter les produits qui y sont disponibles.

Malgré cela, beaucoup d'entre eux trouvent que la pénurie, qui d'après eux n'existe pas dans les villages, est le côté négatif de la vie en ville. En ville, répètent-ils toujours, on doit payer pour tout et l'argent manque pour tout. De façon implicite, cela signifie aussi que le fait d'avoir à payer encore et toujours n'apporte aucune contrepartie sous forme de droits ou de statut. À leurs yeux, l'indépendance n'a pas fonctionné exactement comme ils l'espéraient : les clôtures qui entourent les maisons sont la preuve la plus visible de l'incapacité des gens à recréer en ville une fraternité urbaine ou une grande société de hameau.

D'une certaine façon, la ville sert de test, ou de limite, à l'idéologie du partage à la demande qu'on trouve au hameau. Qui plus est, notons que même si les quartiers de Port-Vila et les maisonnées sont structurés en fonction de l'idée bien rurale de l'entraide mutuelle et du partage, ces quartiers n'ont pas établi pour autant des relations d'échange avec les autres quartiers, comme c'est le cas dans les zones rurales. La seule trace qui reste de la relation intérieur-extérieur propre à la vie rurale est que les maisonnées se renferment sur elles-mêmes; mais comme aucun échange avec le monde extérieur n'a été établi, cette forme d'enfermement devient stérile. En cas de décès, de mariage, de cérémonie de circoncision ou de conflit, les cérémonies rituelles appropriées seront organisées en relation avec les communautés rurales auxquelles les gens appartiennent, ou avec d'autres communautés urbaines avec lesquelles on partage une origine commune. Les échanges cérémoniels entre groupes de voisinage sont très rares. En un sens, les citadins ont transposé en ville une partie de la structure sociale du village soit le partage interne, mais ils n'ont pas transposé l'échange avec l'extérieur. Jusqu'à un certain point, les chefs nommés dans chaque quartier peuvent remédier à la situation en négociant des échanges entre quartiers en cas de conflits, ou si jamais des jeunes filles tombent enceintes par exemple. Mais il est difficile de négocier ces échanges car l'appartenance à une île d'origine interfere avec l'appartenance à 
un quartier et prend toujours le dessus sur cette dernière. La structure villageoise n'est importée qu'à moitié en ville, avec le résultat important suivant : si le partage à la demande existe bien au sein d'un groupe, il n'existe par contre que peu de façons de négocier autonomie et statut individuel. Le partage à la demande et le prélèvement (taking) envahissent toutes les dimensions de statut personnel : aucun aspect de la personne n'est protégé contre le prélèvement. Ceci donne toute sa signification à l'expression stikim nek. L'individu n'a que peu de protection contre les saignées [matérielles et financières] et la construction de clôtures ne fait qu'aggraver le problème.

\section{La maisonnée urbaine comme unité de partage à la demande}

J'ai essayé plus haut d'esquisser la façon dont le partage à la demande fonctionne dans un petit groupe social où les ressources disponibles sont abondantes. En effet, le hameau fonctionne comme une formation sociale où tout le monde a le droit de prendre et de consommer la nourriture des autres. Dans un quartier urbain, la maisonnée, que l'on appelle localement la " cour ", fonctionne comme une extension implicite de ce système. Si vous vivez dans une maisonnée, vous pouvez utiliser tous les produits de consommation qui s'y trouvent. C'est un principe et un impératif moral. J'ai établi plus haut le lien qui existe entre ce principe et la protection presque paranoïaque de la maisonnée. Étant donné que de nombreuses maisonnées font face à toujours plus de précarité, les gens limitent de plus en plus leur taille et leur accès.

Les gens utilisent l'idée de la saignée et la métaphore du stikim nek pour traduire l'importance du danger interne que le partage à la demande représente pour la maisonnée. Dans différents endroits du Vanuatu, cette idée est exprimée par la présence d'un sorcier envieux au sein du groupe, qui examinerait tous les biens et les produits et les convoiterait pour répondre à l'égoïsme manifesté par quelqu'un. Le danger représenté par la saignée, comme dans le cas du sorcier cannibale, c'est qu'elle vide la maisonnée urbaine de l'intérieur vers l'extérieur. Les gens remarquent qu'il leur manque toujours de l'argent ou de la nourriture et expliquent cela en disant que quelqu'un de la maisonnée s'en prend à ses ressources. Par conséquent, les gens limitent l'accès à leur foyer : le magasin domestique sert donc à empêcher précisément qu'un personnage interne à la maison ne s'attaque à ses ressources. Même si la maisonnée n'est pas visiblement ou directement la proie des violeurs ou des voleurs, elle fait l'objet de nombreuses attentions et pressions imaginaires, comme celles venant de forces prédatrices poten- tiellement dangereuses, à la recherche de substances humaines, de chair et de sexualité. L'installation de clôtures et de panneaux d'avertissement reflète cette vision fantasmagorique des choses plutôt que l'existence réelle de crimes, de violence ou de vols. Un élément très important de cette fantasmagorie est l'envie (jelas en bislama). Au sein d'un éthos généralisé d'égalité et de partage, l'envie est devenue une cause de terreur car elle déclenche des forces maléfiques qui mettent en péril la santé et les propriétés. Le voisin qui observe votre propriété, votre femme, vos enfants, votre nouveau moteur hors-bord ou votre nouvelle télévision, peut déclencher une force du mal (demon en bislama) qui a le pouvoir de vous posséder, de vous manger ou de vous détruire. À Port-Vila, et comme le montrent Eriksen et Rio (2017), jelas et demon sont de nos jours les causes de maladie ou de malheurs les plus souvent évoquées. On peut peut-être expliquer cela comme un phénomène de nivèlement existant en regard de l'impératif moral du partage, et impliquant le don et le transfert de toutes ses possessions aux autres membres de la communauté familiale. L'intention du partage est bien d'exercer un poids moral sur les autres ; jelas est l'expression de la demande faite aux autres de partager ce qui leur appartient. On comprend alors que le partage à la demande puisse être conçu, en quelque sorte, comme une force maléfique. D'ailleurs, un des thèmes majeurs développés par l'Église évangélique est de limiter le partage à la demande. C'est dans ce contexte d'inquiétude causée par l'envie et le partage à la demande que les maisonnées de Port-Vila se développent. Et c'est ce qui rend le magasin domestique intéressant.

On peut de nouveau faire une comparaison intéressante avec les données de Rodolfo Maggio à Honiara. Dans le quartier où il habitait, le magasin domestique faisait figure de sanctuaire contre la convoitise, la curiosité et le partage à la demande :

«Au lieu d'acheter au détail des marchandises dans le magasin du coin de la rue, un nombre croissant de résidents de Gilbert Camp achètent aux marchands en gros un stock de nourriture en boîte et les vendent aux membres de leur famille, même à ceux qui font partie de leur foyer, et souvent à eux-mêmes. Par exemple, Marc, le chef d'une famille évangélique, achète une quantité de boîtes de thon et les entrepose dans la maison. À chaque fois que sa femme, Jodie, veut une boîte, elle ne peut pas la prendre simplement sur l'étagère, elle doit la payer. Marc insiste pour qu'elle fasse cela pour tous les repas qu'elle prépare, sans exception. Quand elle n'a pas d'argent, elle inscrit sa dette dans le famili kaonbuk, littéralement le livre de dettes de la famille. Il faut noter que Marc inscrit avec précision autant ses propres dettes que celles de sa femme. Toutefois, la question ici n'est pas que Marc fait un profit en vendant à sa femme au prix du marché. Comme le confirme Jodie, ils ont décidé qu'elle lui achète les produits et qu'il se les achète aussi. " (Maggio, 2016 : 8) 
La moindre demande de partage venant d'un mari, d'un frère ou d'une sœur est traitée comme une demande d'achat. Si tu veux des cigarettes, du sucre, des piles électriques, des unités pour le téléphone portable, tu dois les payer.

Nous avons la chance de pouvoir bénéficier des observations d'Overing sur le cas amazonien (Overing, 1992). Sinon, nous pourrions comprendre tout ceci comme étant le résultat d'une aliénation complète ou d'un individualisme possessif. Comme elle, je pense que nous devons tenir compte du fait que les gens considèrent les magasins comme un lieu d'égalité, de proximité et de communauté. De façon générale, l'histoire du magasin à Port-Vila indique que celui-ci rappelle la plantation et le jardin potager pour deux raisons : d'abord, il s'agit d'un espace plus proche du partage à la demande que de l'échange, et ensuite, cet espace est libre de tous les aspects du stikim nek et de la sorcellerie. Tant que ce sont les marchands chinois qui font tous les profits et qui détiennent le capital, l'argent ne fait plus partie des causes d'envie. Avec un panonceau indiquant que la maison ne fait pas crédit car c'est mauvais pour les affaires, le magasin domestique se présente alors comme un service rendu au voisinage immédiat. Acheter à ce magasin équivaut donc à respecter le partage entre les membres de la parenté, même si cela se fait par l'intermédiaire d'un établissement qui empêche et interdit toute forme agressive de prélèvement. La mise en place d'un magasin domestique est conçue comme un service rendu aux membres de la maisonnée. Ils n'ont plus besoin de parcourir de grandes distances pour se procurer des unités de téléphone, du pain ou du thé puisqu'ils peuvent maintenant se les procurer chez des amis ou des proches en qui ils ont confiance. Cela ne signifie pas pour autant la fin du partage à la demande. Il s'agit plutôt de l'institutionnalisation d'une nouvelle forme de ce type de partage qui désormais ne met plus l'accent sur le fait de prendre, mais plutôt sur le fait de partager, sous une forme pure et monétaire.

\section{Conclusion}

Le grand nombre de clôtures et le confinement des maisons signifient que la ville de Port-Vila se disloque et se divise clairement selon des lignes de cohabitation. Je pense que cet enfermement est dû à un processus social défini selon le principe du partage, mais sans possibilité d'échange cérémoniel ou d'obtention d'autonomie ou de statut personnel. La protection de la maisonnée contre une forme de prélèvement ou de partage à la demande, étendue et universelle - et où tout le monde pourrait prendre le bien des autres - projette sur la ville l'ombre sombre de la paranoïa, alors que la ville aurait pu être l'aboutissement joyeux du mouvement d'indépendance.

A ce propos, je pense que Chris Gregory a mis le doigt sur quelque chose d'important dans un article portant sur l'économie domestique en Inde (2009 : 152). Gardant à l'esprit le modèle de Polanyi sur les processus institués, il pose la question suivante : lors de l'urbanisation, est-il possible aux "processus non institués » de prendre le dessus ? Après tout, il n'est pas toujours facile de transposer au milieu urbain les institutions coutumières issues du milieu rural. À Port-Vila, les gens découvrent que les quartiers ne sont pas formés selon une "façon de vivre mélanésienne » et qu’il n'est pas possible non plus d'y maintenir le partage ou l'échange comme paramètres généraux. En ville, tous ceux qui ne sont pas issus de votre hameau sont en fait des étrangers et des prédateurs possibles de vos avoirs, de vos substances et de votre sexualité. Ces citadins ne sont pas des donneurs de vie et ne peuvent donc pas être approchés dans des échanges cérémoniels. La vie urbaine est plutôt construite sur l'idée du hameau comme principe général. La maisonnée urbaine, elle, a gardé de la vie sociale du hameau l'idée instituée du prélèvement et l'a transformée en un processus non institué : l'achat. Puisque le prélèvement ne peut plus être maintenu en ville, on espère que l'achat sera la solution de remplacement. Acheter est une importation du monde des blancs et des marchands chinois, et comme ce n'est pas une pratique instituée dans la vie sociale du Vanuatu, acheter peut servir de véhicule institué pour purifier la maisonnée urbaine de ses démons. Mais on peut craindre que l'attention portée ainsi aux intérieurs des maisonnées et à la négligence des relations entre maisonnées et voisinages contribuera en fait à l'augmentation des animosités, des hostilités et de l'envie, et feront de Port-Vila une ville où la vie est encore plus difficile.

\section{Remerciements}

Cet article a été préparé pour la conférence de la Société européenne des Océanistes tenue à Bruxelles en 2015. Je remercie les participants à cette session pour leurs commentaires et les organisateurs, Lamont Lindstrom et Christine Jourdan, pour les commentaires détaillés qu'ils ont faits sur cet article. Je remercie aussi Laurent Dousset pour sa lecture d'une version préliminaire de ce texte, ainsi que les lecteurs anonymes du JSO pour leurs utiles commentaires. Mes remerciements aussi à Christine Jourdan qui a traduit en français ce texte écrit en anglais. 


\section{BIBLIOGRAPHIE}

ERIKSEN Annelin, 2008. Gender, Christianity and Change in Vanuatu. An analysis of Social Movements in North Ambrym, London, Ashgate.

Eriksen Annelin et Knut Rio, 2017. Demons, Devils and Witches in Pentecostal Port Vila: On changing cosmologies of Evil in Melanesia, in K. Rio, M. MacCarthy and R. Blanes (eds), Pentecostalism and Witchcraft in Melanesia and Africa, London, Palgrave.

GeLl Alfred 1992. Inter-tribal commodity barter and reproductive gift exchange in old Melanesia, in C. Humphrey and S. Hugh-Jones (eds), Barter, Exchange and Value, Cambridge, Cambridge University Press, pp. 142-169.

Gregory Chris, 2009. What happened to householding, in C. Hann and K. Hart (eds), Market and Society, Cambridge, Cambridge University Press, pp. 133-160.

Jimenez Alberto Corsin et Rane Willerslev, 2007. An anthropological concept of the concept': Reversibility among the Siberian Yukaghirs, JRAI 13, pp. 527-544.

LAYARD John, 1942. Stone men of Malekula, London, Chatto and Windus.

Maggio Rodolfo, 2014. "Honiara is hard". The Domestic Moral Economy of the Kwara'ae people of Gilbert Camp, Solomon Islands, Phd Thesis, University of Manchester.

—, 2016. 'My wife converted me': Gendered values and gendered conversion in Pentecostal households in Honiara, Solomon Islands, The Australian Journal of Anthropology 27 (2), pp. 168-184.

Overing Joanna, 1992. Wandering in the market and the forest, in R. Dilley (ed.), Contesting Markets, Edinburgh, Edinburgh University Press, pp. 180-203.
Peterson Nickolas, 1993. Demand-Sharing: Reciprocity and the pressure for generosity among foragers, American Anthropologist 95 (4), pp. 860-874.

Polany Karl, 1957. The economy as instituted process, in K. Polanyi, C. Arensberg and H. Pearson (eds), Trade and Market in Early Empires, New York, The free Press, pp. 243-270.

Riches David, 2000. The holistic person; or, the ideology of egalitarianism, JRAI 6, pp. 669-685.

Rio Knut, 2002. The sorcerer as an absented third person. Formations of fear and anger in Vanuatu, Social Analysis 46, pp. 129-154.

—, 2007a, The Power of Perspective, Oxford, Berghahn Books.

—, 2007b. Denying the Gift: aspects of the gift and its counter-prestation in a Vanuatu society, Anthropological Theory 7( 4), pp. 449-470.

—, 2018 (à paraître). 'Witchcraft' and 'Sorcery' in Melanesia, in E. Hirsch and W. Rollason (eds), The Melanesian World, London, Taylor \& Francis.

SaHlins Marshall, 1972. Stone Age Economics, Chicago, Chicago University Press.

Servy Alice, 2017. "AIDs is here!” Prévenir les infections sexuellement transmissibles à PortVila, Vanuatu', thèse de doctorat, Marseille École des hautes études en sciences sociales.

WIDLOK Thomas, 2013. Sharing. Allowing others to take what is valued, $\operatorname{HAU} 3$ (2), pp. 11-31.

Woodburn James, 1998. 'Sharing is not a form of exchange': An analysis of property sharing in immediate-return hunter-gatherer societies, in C. M. Hann (ed.), Property relations: Renewing the anthropological tradition, Cambridge, Cambridge University Press pp. 48-63. 